\title{
Turismo de surf: revisión de nuevas líneas y temáticas de investigación (2012-2018)
}

\section{Surf tourism: a review of new lines and topics of research (2012-2018)}

Luis Valencia iD, Universidad Autónoma del Estado de México, México alberto valenciach@hotmail.com

Maribel Osorio García iD, Universidad Autónoma del Estado de México, México maribelosorio2@gmail.com

Rocío del Carmen Serrano Barquín (iD, Universidad Autónoma del Estado de México, México rocioserba14@gmail.com

\section{RESUMEN}

El turismo de surf es un fenómeno de gran relevancia a nivel mundial; millones de surfistas se desplazan a diversos destinos con la finalidad de montar la ola perfecta, mejorar sus habilidades, aprender el deporte o simplemente apreciarlo. Sin embargo, aun cuando se han reconocido las características particulares de este nicho de turismo en Iberoamérica, poca atención ha recibido como fenómeno social por parte de la Academia y, por lo tanto, la revisión de su estado de conocimiento ha sido limitada. El presente documento tiene como objetivo presentar las líneas de investigación relacionadas con el turismo de surf durante el periodo 2012-2018, a través de un análisis de contenido aplicado a artículos científicos publicados tanto en inglés como en español. Entre los principales resultados destacan cinco líneas de investigación: 1) El surfista; 2) Turismo de surf, desarrollo local y sustentabilidad; 3) Impactos del turismo de surf; 4) Identidad y género en el turismo de surf; e 5) Historia del surf y stakeholders, las cuales son descritas en este estudio. Cabe señalar que la revisión realizada también permitió identificar autores, universidades y países que han abordado temas relacionados, así como aquellos vacíos de conocimiento que aún persisten sobre este tópico de actualidad.

Palabras claves: turismo de surf; revisión de la literatura; análisis de contenido. 


\section{ABSTRACT}

Nowadays surf tourism is a phenomenon of great relevance worldwide, in which millions of tourists move in order to ride the perfect wave, improve their skills, learn the sport or simply appreciate it. Although the particular characteristics of this tourism niche have been recognized, little attention has been received by the academy as a social phenomenon and therefore little is known about the state of knowledge surrounding surf tourism. In this sense, the main goal of this document is present the research lines that have emerged around surfing tourism within the 2012-2018 period through a content analysis of the scientific articles published in both, English and Spanish. From the analysis, five main lines were obtained: 1) The surfer; 2) Local development and sustainability; 3) Impacts of surf tourism; 4) Identity and gender; 5) History of surfing and stakeholders. This review also allowed to identify authors, universities and countries that have addressed to a greater extent issues related to surf tourism, as well as, those knowledge gaps that still persist in this field of research in.

Keywords: surf tourism; literature review; content analysis.

\section{INTRODUCIÓN}

El surf es una actividad que consiste en el aprovechamiento directo de la naturaleza; el viento, el agua y las olas, y que reside en deslizarse de pie sobre una tabla hasta que rompe en la costa (Esparza, 2011). En la actualidad es un deporte de gran relevancia a nivel mundial que es practicado en los cinco continentes del planeta; tal es su importancia, que en el 2020 debutará como uno de los nuevos deportes que formarán parte de los Juegos Olímpicos (JJOO) que se llevarán a cabo en Tokio, Japón según la International Surfing Assotiation (ISA, 2018). Entre las razones principales de dicha expansión paulatina, se encuentran el lanzamiento de las películas Gitget (1959) y The Endless Summer (1966) en la década de los 60's, según señalan Martin y Assenov (2012), pues ambas muestran parte del estilo de vida surf de ese entonces que hasta nuestros días impera: el joven libre montando las olas. De esta forma el surf se convierte en un deporte que tiene la capacidad de mover a grandes cantidades de surfistas alrededor del globo terráqueo en busca de la ola perfecta para montar (Buckley, 2002), destacando en dicha actividad países como Estados Unidos, Australia, Brasil, Portugal, entre otros (Hritz y Franzidis, 2018).

Si bien la práctica del surf alrededor del mundo se ha vuelto algo común, al día de hoy no existe un acuerdo entre los investigadores sobre cómo definir el turismo de surf. Por ejemplo, Dolnicar y Fluker (2003) señalan que el turismo de surf debe ser entendido como "el acto de personas viajando a destinos nacionales [...] o internacionales, donde existe participación activa en el deporte del surf [...]" (p. 4), mientras que Ponting (2008) reconoce la participación activa en la práctica del turismo de surf, dejando fuera de su conceptualización a aquellos que viajan a aprender dicho deporte, apreciarlo o como acompañantes de un surfista. Por su parte, Buckley (2002) desde un enfoque estadístico económico lo entiende como aquel desplazamiento mayor a 40 kilómetros de la residencia habitual, en el cual los surfistas 
duermen como mínimo una noche en el lugar visitado, característica en la que coincide con Ponting (2008).

El Tourism New South Wales (2009, p.3), señala que el turismo de surf comprende aquella actividad que toma lugar a 40 kilómetros o más del lugar de residencia de la persona, donde el surf se convierte en el primer propósito de viaje; sin embargo, también se reconoce que ese viaje puede estar motivado por un evento relacionado con dicha práctica, lo que resulta ser una característica nueva que se adiciona a las definiciones anteriores.

Con base en todo lo anterior, para los autores del presente texto el turismo de surf comprende a todos aquellos viajes realizados por los surfistas activos (expertos, practicantes y novatos) y pasivos (espectadores y acompañantes) a destinos diferentes de su entorno habitual donde el surf en cualquiera de sus modalidades resulta ser la motivación principal del viaje y donde existe por lo menos una noche de pernocta, sin importar la distancia recorrida.

Al día de hoy no existe una cifra exacta sobre el número de personas que practican surf; y según Esparza (2015), esto se debe a que muchos surfistas no se encuentran registrados en alguna federación, o en algunos casos, practican el deporte de manera esporádica o irregular, dificultando su identificación como turistas; sin embargo, su crecimiento no se puede negar. Tan sólo en la década del 2000 se hablaba de más de 10 millones de turistas surfistas viajando alrededor del mundo (Buckley, 2002), pero en 2016 esa cifra llegó a 23 millones, según señaló Fontrodona (2016) a partir de datos de la International Surfing Assotiation.

Pese a dicho crecimiento, en diversas investigaciones (Towner, 2016b; Mills y Cummins, 2013; Dolnicar y Fluker, 2003) se señala que el principal interés que el turismo de surf ha recibido por parte de la Academia y los gobiernos de algunos países es de carácter económico. De esta forma, se considera que se está dejando de lado el aspecto sociológico del surf, al mismo tiempo que se pasan por alto sus implicaciones de carácter cultural en la sociedad contemporánea (Buckley, 2002; Hritz y Franzidis, 2018).

Dos investigaciones, que pueden ser consideradas como las más importantes para entender parte del desarrollo del turismo del surf a partir de la academia, son las realizadas por Martín y Assenov (2011; 2012), pues ambas dan muestra de los temas de mayor interés por parte de los investigadores, gobiernos, ONG's y otros actores. En la publicación del 2012, que comprende el periodo 1997-2011, los autores identificaron poco más de 5000 documentos entre tesis, conferencias, reportes de ONG's, artículos, capítulos de libros, literatura gris, entre otros, que hablan sobre el surf. De particular importancia resulta mencionar que únicamente se identificaron 42 artículos científicos y de esos, sólo 23 tocaban temas exclusivos de turismo de surf; cifra que representa la publicación de 1.5 artículos por año. Es por tal motivo que ambos autores reconocen al turismo de surf como un campo de investigación que se encuentra en su génesis, siendo países como Australia y Estados Unidos punta de lanza en este campo de estudio. Por lo anterior, no resulta extraño que la mayoría de las publicaciones identificadas por los autores hayan sido en inglés, aunque los casos de estudios no sean únicamente de los dos países antes mencionados. 
Tomando en consideración el crecimiento del turismo de surf y, a modo de dar seguimiento a los trabajos realizados por Martín y Assenov (2011; 2012), el presente estudio tiene como objetivo central identificar las líneas de investigación en torno al turismo de surf durante el periodo 2012-2018, a partir de la revisión de los artículos científicos publicados dentro de ese lapso, tanto en inglés como en español. Se ha decidido tomar en cuenta las publicaciones en español porque durante la investigación de Martín y Assenov (2012) únicamente se contabilizaron 10 publicaciones (tesis, artículos, capítulos de libros, etc.) a pesar de que países como Perú, México, Chile, España y Argentina son reconocidos a nivel mundial por poseer buenos lugares para la práctica de dicho deporte.

\section{METOdOLOGÍA}

Se consultaron las bases de datos de Google Scholar, Science Direct, Redalyc, Dialnet, Scielo, y Taylor \& Francis; aunque en algunos casos los artículos fueron buscados de manera independiente, sobre todo publicaciones en español. La búsqueda se realizó a partir de las palabras como "surf" y "tour" utilizadas por Martin y Assenov (2012), más "surfing" "surfing tourism" y "turismo de surf". En total se identificaron 107 artículos que correspondían al periodo seleccionado, más el artículo de Martin y Assenov (2012), pero este último no fue tomado en cuenta para el cumplimiento del objetivo central de esta investigación en la medida en que se tomó como punto de partida para la elaboración de la misma.

Del total de artículos encontrados, se realizó una depuración inicial bajo el criterio de eliminación de aquellos documentos que, dentro de su título, resumen o abstract, o palabras clave (key words) no hablaran sobre el surf, el turismo de surf, el surf como deporte, destinos de surf, o bien, los turistas surfistas. Como segundo criterio, se eliminaron los artículos incluidos en reseñas de libros, o que correspondieran a evaluaciones de programas o eventos de surf; y como tercer criterio de eliminación, se ignoraron aquellos textos que abordaban temas que no estuvieran relacionados con aspectos sociales, culturales, económicos o políticos relativos al surf (propios de las Ciencias Sociales). Por ello no se tomaron en cuenta los documentos relativos a factores geofísicos, condiciones oceánicas y olas; surf en piscinas artificiales, marcas de ropa de surf, enfermedades relacionadas con el surf, heridas; fabricación de tablas para surf, aspectos relacionados a ceremonias fúnebres, salvavidas, así como estudios relacionados con algún segmento de practicantes de surf que no estuviera relacionado con el turismo.

A partir de dicha depuración, con base en los criterios señalados, se consideraron un total de 81 artículos que cumplían con los requerimientos antes mencionados: que estuvieran íntimamente relacionados con el turismo de surf, y que estuvieran relacionados con temas correspondientes al área de conocimiento de las Ciencias Sociales. Los textos encontrados fueron ordenados por año y quedaron distribuidos como se muestra en la tabla 1. 
Tabla 1. Número de artículos publicados por año

\begin{tabular}{|c|c|}
\hline AÑo DE PUBLICACIÓN & $\begin{array}{c}\text { NÚMERO DE ARTíCULOS } \\
\text { POR AÑO }\end{array}$ \\
\hline 2018 & 18 \\
\hline 2017 & 12 \\
\hline 2016 & 13 \\
\hline 2015 & 11 \\
\hline 2014 & 13 \\
\hline 2013 & 8 \\
\hline 2012 & 6 \\
\hline Total de artículos & 81 \\
\hline
\end{tabular}

Elaboración propia.

A través de un análisis del discurso (lectura del resumen o abstract, palabras claves y contenido) de los artículos seleccionados, se identificaron líneas temáticas generales en las cuales se integraron las publicaciones revisadas.

El agrupamiento de los artículos por año, permite identificar cómo del 2012 al 2018 ha habido un incremento paulatino en cuanto al número de publicaciones sobre turismo de surf, las 42 publicaciones identificadas por Martin y Assenov (2012) correspondientes al periodo 1997-2011; demostrando de esta forma que el turismo de surf ha adquirido mayor interés por parte de los investigadores. Asimismo, se puede observar que es a partir del 2014 cuando se observa un incremento considerable en cuanto al número de artículos publicados.

En relación con las revistas donde la mayoría de los autores publican sus trabajos destacan principalmente: Annals of Leisure Research, Tourism Planning \& Development, Annals of Tourism Research, Asia Pacific Journal of Tourism Research, Ocean \& Coastal Management y Journal of Sustainable Tourism. Haciendo una comparación entre las principales revistas identificas por Martin y Assenov (2012), se encontró que hay coincidencia en tres revistas (Tourism Planning \& Development, Annals of Tourism Research, Journal of Sustainable Tourism), que dichos autores identificaban con sólo una publicación en ese entonces.

Por su parte, revistas como Annals of Leisure Research, Asia Pacific Journal of Tourism Research y Ocean \& Coastal Management que no fueron identificadas por Martin y Assenov (2012), actualmente aparecen como las que encabezan la lista, al menos en este estudio. La tabla 2 muestra el concentrado de todas las revistas identificadas y el número de artículos publicados en ellas durante el periodo de estudio, 2012-2018. 
Tabla 2. Revistas identificadas a través de los artículos seleccionados

\begin{tabular}{|c|c|}
\hline TÍTULO DE LA REVISTA & $\begin{array}{l}\text { NÚMERO DE ARTÍCULOS } \\
\text { PUBLICADOS POR } \\
\text { REVISTA }\end{array}$ \\
\hline Annals of Leisure Research & 6 \\
\hline Tourism Planning \& Development & 5 \\
\hline $\begin{array}{l}\text { Asia Pacific Journal of Tourism Research; Annals of Tourism Research; Ocean \& } \\
\text { Coastal Management }\end{array}$ & 4 \\
\hline Journal of Sustainable Tourism; Journal of Sport \& Tourism & 3 \\
\hline $\begin{array}{l}\text { Leisure Studies; Leisure Sciences; European Journal of Tourism Research; Journal } \\
\text { of Tourism Research; Materiales para la Historia del Deporte; (Ricyde) Revista } \\
\text { Internacional de Ciencias del Deporte; Estudios Turísticos }\end{array}$ & 2 \\
\hline $\begin{array}{l}\text { Journal of Sports and Physical Education (IOSR-JSPE); Journal of Destination } \\
\text { Marketing \& Management; Surf Spot, BMIJ; Tourism Review; European Planning } \\
\text { Studies; Journal of Ecotourism; Journal of Tourism and Cultural Change; } \\
\text { International Journal of Spa and Wellness; Journal of Sport \& Social Issues; } \\
\text { International Review for the Sociology of Sport; Australian Journal of Maritime } \\
\text { \& Ocean Affairs; Pelotas; Society and Leisure; Journal of Park \& Recreation } \\
\text { Administration; Journal of Media \& Cultural Studies; Marine Policy; Journal } \\
\text { of Hospitality and Tourism Management; Sport, Education and Society; Sport } \\
\text { Management Review; Journal of Sport and Social; Int. J. Tourism Anthropology; } \\
\text { Journal of Spatial and Organizational Dynamics; Tourism Management; Journal of } \\
\text { African CulturalStudies; Progress in Development Studies; Journal of China Tourism } \\
\text { Research; Coastal Management; Journal of Sport Management; Consumption } \\
\text { Markets \& Culture; Anthopology in Action; Tourism in Marine Environments; } \\
\text { Journal of Australian Studies; (Ricyde) Revista Internacional de Ciencias del } \\
\text { Deporte; Revista Turismo \& Desenvolvimiento; Motriz, Rio Claro; TELOS. Revista } \\
\text { de Estudios Interdisciplinarios en Ciencias Sociales; Romanica Olomucensia; } \\
\text { Cuadernos de Turismo }\end{array}$ & 1 \\
\hline
\end{tabular}

Elaboración propia.

A partir de la agrupación de los autores dentro del periodo estudiado, se identificó que la mayoría de los artículos fueron realizados entre dos o más investigadores de una misma institución, con un total de 25. En cuanto a la colaboración entre investigadores de un mismo país, pero de diferente universidad, en total se identificaron 14 publicaciones. De particular importancia resulta mencionar que de las 81 publicaciones identificadas, 13 se realizaron entre investigadores de distintas universidades, 2 de diferentes países y sólo una conjugando la participación de tres naciones. Destaca la participación conjunta que existe entre investigadores de universidades de Estados Unidos y Australia.

También se identificó que sólo 26 artículos fueron escritos por un solo autor, destacando en este aspecto Nick Towner (del Auckland Institute of Studies de Nueva Zelanda) con 5 artículos, seguido por Daniel Esparza (Universidad Palacky de Olomouc, de la República Checa); éste último por ser de los pocos investigadores que han escrito artículos en español y que además ha centrado su trabajo en aspectos relacionado con la historia del surf en determinados destinos. La tabla número 3 concentra los nombres de los autores que han publicado tres o más artículos relacionados con el surf, así como a la universidad a la que están adscritos. 
Tabla 3. Autores que más artículos han publicado sobre Turismo de Surf

\begin{tabular}{|l|l|c|}
\hline \multicolumn{1}{|c|}{ AUTOR } & \multicolumn{1}{c|}{ UNIVERSIDAD } & $\begin{array}{c}\text { NÚMERO DE } \\
\text { ARTÍCULOS }\end{array}$ \\
\hline Nick Towner & Auckland Institute of Studies, Nueva Zelanda & 9 \\
\hline Lindsay E. Usher & Old Dominion University, Estados Unidos & 9 \\
\hline Jess Ponting & San Diego State University, Estados Unidos & 7 \\
\hline Daniel Esparza & Universidad Palacky de Olomouc, República Checa & 5 \\
\hline Steven Andrew Martin & Prince of Songkla University, Tailandia & 3 \\
\hline Edwin Gómez & Old Dominion University, Estados Unidos & 3 \\
\hline Carla Barbieri & North Carolina State University, Estados Unidos & 3 \\
\hline Deborah Kerstetter & Pennsylvania State University, Estados Unidos & 3 \\
\hline Danny O'Brien & Bond University, Australia & 3 \\
\hline Mark Orams & Auckland University of Technology, Nueva Zelanda & 3 \\
\hline
\end{tabular}

Elaboración propia.

La tabla anterior permite vislumbrar que Estados Unidos es el país que más investigaciones ha realizado en torno al turismo de surf o al surf mismo; si bien en la tabla aparecen países como Nueva Zelanda, Australia, República Checa y Tailandia, los dos últimos como tal no son países que destaquen en cuanto al número de publicaciones en relación al estudio. Por el contrario, Australia, Nueva Zelanda, Portugal, Reino Unido y España, sí tienen gran participación en el número de publicaciones. Pero la mayor parte de ellas se concentra en Estados Unidos y Australia, datos que también coinciden con los identificados por Martin y Assenov (2012), pero a la inversa, pues durante el periodo 1997-2011, las universidades australianas encabezaban la lista.

Para la identificación de las líneas de investigación se llevó a cabo un análisis del discurso de los documentos elegidos, el cual consiste en una estrategia aplicada en las Ciencias Sociales en la que el investigador "se enfrenta a textos, o a signos de diversa naturaleza (no necesariamente lingüísticos), que requieren ser leídos para su correcta interpretación" (Santander, 2011 , p. 208). En el siguiente apartado, se presentan las líneas de investigación identificadas y las aportaciones al conocimiento que se realizan en los artículos identificados relacionados con el turismo de surf.

\section{RESULTADOS: LÍNEAS DE INVESTIGACIÓN DEL TURISMO DE SURF}

A partir del análisis de los artículos seleccionados se identificaron cinco líneas de investigación: 1) El surfista; 2) Desarrollo local y sustentabilidad; 3) Impactos del turismo de surf; 4) Identidad y género; 5) Historia del surf y stakeholders.

La línea de investigación relacionada con el surfista, comprende todas las publicaciones que tienen relación con la identificación del perfil del turista surfista: sus actitudes hacia el medio ambiente y motivaciones de viaje. La segunda línea de investigación, la de desarrollo local y sustentabilidad, toma en cuenta temas como políticas y estrategias de planificación y 
desarrollo del turismo de surf, participación local en el turismo de surf, y gestión de zonas costeras para la preservación de los puntos de surf (spots).

La tercera línea de investigación identificada, agrupa los estudios de impactos del turismo de surf, en la que se incluyen temas como localismo e impactos económicos, ambientales y socioculturales. La cuarta línea concentra las investigaciones sobre identidad y género, la cual comprende temas como el papel y reto de las mujeres surfistas, la identidad a través de surf, entre otros. Finalmente, la última línea abarca, por un lado, investigaciones relacionadas con la historia y desarrollo del surf en algunos destinos, y por el otro, aquellas que tomarán en cuenta a todos aquellos interesados en el turismo de surf en un determinado contexto. El resto de los artículos que por su tema central no podían ser considerados dentro de una de las líneas anteriores, son presentados de manera conjunta.

\subsection{Descripción de las líneas de investigación}

La línea de investigación que mayor atención ha recibido por parte de los investigadores que abordan el surf como campo de estudio, es la del surfista: de los 81 artículos identificados, 21 (25. 92\%) estuvieron relacionados con este tema. Aunado a lo anterior, resulta importante mencionar que 15 de esas publicaciones fueron de corte cuantitativo, utilizando como técnica principal para recabar información la encuesta.

A partir de la revisión de los 21 documentos identificados que conforman la presente línea, los artículos se agruparon en tres grandes temáticas; 1) Apego al lugar y conciencia ambiental; 2) perfil del turista surfista; 3) motivaciones del surfista. Otras temáticas específicas fueron: la participación recreativa del surfista y el bienestar (Tien-Ming y Chiang-Chuan, 2015), los significados de viajes para los surfistas (Barbieri et al., 2014), estudios relacionados con las experiencias de viaje (Brochado et al., 2018), comparación de servicios entre operadores turísticos en el turismo de surf (Ponting, 2014) y finalmente, construcción del espacio turístico en relación con el surf a partir de los mitos -Nirvana- (Ponting y McDonald, 2013).

\subsubsection{El surfista}

La categoría en la que más publicaciones se identificaron gira en torno a la consciencia ambiental y el apego del surfista a los puntos de surf o también llamados spots, y que se refieren a todos aquellos lugares que cuentan con las condiciones necesarias para practicar alguna modalidad de surf; esto no es de extrañar, pues diversos investigadores han destacado que este segmento de turistas es consciente de la fragilidad ambiental y, por lo tanto, buscan su conservación. Entre los autores que han trabajado esta temática se encuentran Larson, et. al. (2018) y Frank, et. al. (2015) quienes en sus respectivos estudios tocaron temas relacionados con la conservación, el apego a los lugares visitados y los comportamientos proambientales de los surfistas. A partir de su investigación, Frank et. al., señalan que la mayoría de éstos incluso estaría dispuesto a pagar un impuesto extra que esté destinado al cuidado del medio ambiente. 
Tomando en cuenta el apego a los spots por parte de los surfistas, Reineman y Ardoin (2018) encontraron que algunos surfistas los consideran como parte de su familia y reconocen la importancia de su cuidado. En ese sentido en la investigación realizada por Aaron y Wayne (2013) en Auckland y Otago, Nueva Zelanda, los autores reconocen que los surfistas pueden ayudar al manejo y desarrollo de los spots. Finalmente, a modo de enmarcar lo anterior, el trabajo de D'ambrosio (2017) permite visualizar la importancia que el mar y la playa tienen para el surfista. Esto a través de una comparación con pescadores y biólogos en relación a la percepción y uso de la costa y el mar.

En total fueron 6 los artículos que se encontraron relacionados con estudios del perfil del turista surfista; en su conjunto, dichos estudios buscan comprender quién es el turista surfista en contextos específicos o a nivel internacional. Entre esas investigaciones destacan la de Towner (2016b) en las Islas Mentawai, la de Sotomayor y Barbieri (2016) en el ámbito internacional y teniendo como base el marco del ocio serio, y la de Porter y Usher (2018). En esta misma temática de investigación, se encontró que diversos estudios se han realizado tomando como base el ocio serio y sus seis elementos (Esfuerzo, Ética, Carrera, Identidad, Perseverancia, Beneficios).

Entre esos estudios se encuentra el de Tien-Ming y Sheng-Hshiung (2012) en Taiwan, Barbieri y Sotomayor (2013) y Portugal, et. al. (2017), quienes a través de su estudio comparan las seis cualidades del ocio serio con el comportamiento de viaje y las preferencias de los surfistas. Los resultados confirman que los turistas surfistas muestran altos niveles de ocio serio en sus seis cualidades. Finalmente, se encuentra la investigación de Beaumont y Brown (2015) quienes a través del análisis de la carrera del surf identificaron que ésta comprende etapas compartidas: la etapa nutrida, la etapa competitiva posible, la etapa del viajero de ocio serio, la etapa responsable y la etapa de leyendas.

En cuanto a los estudios relacionados con las motivaciones de los turistas surfistas se identificó el elaborado por Silva, et. al. (2014) en Portugal y el de Usher y Gómez (2017) en Virginia Beach, Estados Unidos, los que demuestran que, el hacinamiento de turistas, constituye un factor que provoca lesiones a los surfistas, conflictos e insatisfacción y, por lo tanto, afecta la motivación de viaje.

Otro estudio identificado es el de Scott y Rogers (2018), el cual demuestra que la contaminación del agua es un factor que incide en la decisión de no viajar por parte de los surfistas, pues la consideran un riesgo para su salud. Por su parte, Kumara, et. al. (2018) en una investigación llevada a cabo en Sri Lanka, identificaron los factores que inciden para que los surfistas de esa comunidad no realicen viajes al exterior. Entre ellos se encuentran la accesibilidad, la conciencia, la falta de recursos y los patrocinadores, entre otros. Finalmente, Mach, et. al. (2018) analizaron cómo el oleaje resulta ser un componente indispensable que marca la estacionalidad en la práctica del turismo de surf.

El grupo de temáticas de investigación que conforman esta primera línea permite apreciar que el turista surfista tiene un apego considerable a los lugares que visita y, por lo tanto, está dispuesto a contribuir con su conocimiento para su protección y cuidado. E incluso, está dispuesto a pagar un impuesto extra para el cuidado de los spots; esto quizás se debe a que 
este segmento es consciente de la fragilidad del ambiente y su estrecha relación con la práctica de dicho deporte. También resulta evidente que diversos son los factores que influyen en la motivación de viaje de este segmento de turistas como son el hacinamiento en los destinos, la calidad del agua y de las olas, los servicios complementarios, entre otros. Por lo tanto, se considera que aquellos destinos que apuesten al desarrollo de este segmento deberían estar conscientes de esos factores y buscar de esta forma no afectar la experiencia de viaje del surfista.

A partir de los artículos presentados se evidencia cómo el ocio, pero principalmente los factores que inciden en el ocio serio, resulta ser un tema que está adquiriendo gran atención por parte de los investigadores. Por lo tanto, constituye una línea de exploración potencial a ser revisada.

\subsubsection{Turismo de surf, desarrollo local y sustentabilidad}

Dentro de esta línea de investigación se incluyeron estudios que estuvieran destinados a la planificación, gestión y uso de los puntos de surf -spots- o de las zonas costeras, así como a la participación de la comunidad en torno a la sustentabilidad, entre otros aspectos afines. En total se identificaron 21 artículos (25.92\%), la mayoría relacionados con la planificación y gestión de los puntos de surf. Resulta importante mencionar que esta línea ha recibido gran atención por parte de los investigadores, desde el estudio realizado por Martin y Assenov (2012).

Entre esos artículos se encuentran el elaborado por O'Brien y Ponting (2013) que busca responder a la pregunta: ¿Cómo se puede gestionar el turismo de surf, para lograr beneficios sostenibles para la comunidad de Papua, Nueva Guinea?; los autores concluyen su investigación señalando que involucrar activamente a los miembros de la comunidad dentro de un marco de colaboración resulta ser la mejor opción. En cuanto al manejo de los puntos de surfo zonas costeras de forma sostenible, se identificaron varios estudios centrales: Towner (2016c) en las Islas Mentawai; Ware (2017) en la Costa Dorada de Queensland; Reineman (2016) en California; Orchard (2017) en Nueva Zelanda y Australia; Usher, et. al. (2016) en Virginia Beach y finalmente Martín y Luque-Gil (2014) en Costal del Sol Occidental, España.

En el primero de ellos se concluyó que la forma en cómo se estaba manejando el turismo de surf en las Islas era inadecuada y que eso se debía en gran parte a la corrupción por parte del gobierno. En el estudio de Ware (2017), los surfistas señalaron que a pesar de que en la Costa Dorada se aplicó el programa de gestión integrada de las zonas costeras, se sigue afectado de manera considerable los puntos de surf por parte del desarrollo aprobado por el gobierno. En torno a la sostenibilidad de los puntos de surf, el estudio de Reineman (2016) reconoce que el conocimiento de los surfistas respecto a las olas, puede contribuir significativamente a su correcta gestión. Por último, el estudio de Orchard (2017) presenta y discute los enfoques innovadores que se han utilizado en Nueva Zelanda y Australia para el cuidado de los puntos de surf, siendo reconocidos a nivel mundial y en algunas ocasiones replicados, mientras que Usher, et. al. (2016), a través de las opiniones de los surfistas estudian las regulaciones de un municipio en torno al surf presentando los aspectos positivos, los negativos y 
sus confusiones, entre otros aspectos. Finalmente, Martín y Luque-Gil (2014) a través de su investigación en Costal del Sol Occidental, proponen una serie de estrategias para diversificar, a través del surf, la oferta turística en el destino tomando como referencia el Plan Qualifica.

Otros trabajos bajo esta temática son el de Jopp, et. al. (2013), Martin y Assenov (2014a, 2014b), y el de Martin y Ritchie (2018). El primero de ellos toma como base el cambio climático para después explicar la aplicación de un modelo de adaptación del turismo de surf en Victoria, Australia (Jopp et al., 2013). Mientras que los otros tres restantes, tienen la característica de utilizar determinados indicadores para evaluar los factores de sostenibilidad en el surf. En este sentido, resulta importante mencionar que los tres trabajos en los que aparece el investigador Andrew Martin obedecen a este tópico.

Bajo el tema de participación local en el turismo de surf se identificaron 7 artículos. El primero de ellos es de Nazlı y Musal (2018), que tuvo como objetivo analizar a través de las percepciones y opiniones de los empleados y visitantes la sostenibilidad en siete centros de surf en Alaçat, Turquía. Por su parte, la investigación de Machado, et. al. (2018), haciendo uso de la participación local obtuvo una lista de indicadores económicos, ambientales y sociales que permitieron elaborar una carta municipal de gestión sostenible del turismo de surf en la región. Por otra parte, en su estudio, Ponting y O'Brien (2015) exploran la política de gestión de capacidad recreativa del turismo de surf en las islas Mentawai, así como sus implicaciones en la economía local. Un estudio que también destaca esta temática es el elaborado por Eka (2012), pues se realiza a partir de la perspectiva del turismo de base comunitario.

Por su parte, Towner (2016a) en las Islas Mentawai investiga el nivel de participación de los residentes locales en el turismo de surf, concluyendo que éstos en su mayoría, han quedado fuera de sus beneficios. De forma contraria, Porter, et. al. (2015) analizan la posibilidad de que el turismo de surf sea una oportunidad para lograr el desarrollo local en las comunidades pesqueras de Filipinas. Bajo esta misma temática de desarrollo local y sustentabilidad fueron realizadas también las investigaciones de Guibert y Taunay (2013) en la Islas Hainan, China y la de Ponting y O’Brien (2014) en las Islas Fiji.

Finalmente destacan tres trabajos, el de Martin y Ritchie (2018), Mach y Ponting (2018) y Rivera (2016). El primero de ellos porque los autores desarrollaron un esquema de ponderación para la planificación del turismo de surf de manera sostenible tomando como destino representativo Phuket, Tailandia y haciendo uso de 27 indicadores sociales, económicos, ambientales y de gobernabilidad. El segundo por su parte, aunque es una investigación teórica, destaca por ser el único documento identificado en relación con la gobernanza en los destinos de surf, mismo que busca incentivar estudios bajo esta temática y que, por lo tanto, resulta ser un área de oportunidad para futuras investigaciones (Mach y Ponting, 2018). Finalmente, el trabajo de Rivera (2016) destaca por ser de los pocos que reconoce la importancia de todos aquellos factores naturales que hacen posible la práctica del surf, al mismo tiempo que señala la importancia de su cuidado y preservación.

En este apartado resulta necesario mencionar que, debido a la particularidad del tema, se ve un incremento de estudios realizados con un enfoque cualitativo apoyados de la observación participante. También es relevante mencionar que las Islas Mentawai fueron el caso de 
estudio en cuatro investigaciones debido a que es un referente a nivel mundial en cuanto a destinos de surf. Asimismo, se destaca el papel de investigador Martin de la Prince of Scogkla University, Tailandia, en tanto que ha trabajado bajo la línea de sostenibilidad todas sus investigaciones (las identificadas en este estudio).

\subsubsection{Impactos del turismo de surf}

Los impactos del turismo en general han sido uno de los temas más abordados por los investigadores; sin embargo, se considera que la mayoría de estos estudios se han centrado en el turismo convencional, dejando de lado qué tipos específicos de turismo generan impactos particulares. En ese sentido, si bien los artículos ubicados en esta línea pudieron haber sido contemplados dentro de la línea anterior -desarrollo local y sostenibilidad- se ha optado manejarlos de forma independiente debido a que ya forman como tal una línea de investigación.

En este sentido, los impactos considerados en este apartado son los económicos, los ambientales y los socioculturales, destacando los últimos. A través del estudio de las relaciones entre los surfistas locales y los surfistas turistas se ha estudiado el localismo; éste debe ser entendido como ese derecho -territorial- y preferencia que los surfistas locales manifiestan tener ante los turistas surfistas al momento de surfear una ola del destino donde viven. De los 11 (13.58\%) artículos considerados dentro de esta línea, cinco presentan como tema central el localismo (Waitt y Frazer, 2012; Martins Bandeira, 2014; Usher y Kerstetter, 2015a, Beaumont y Brown, 2016; Usher y Gómez, 2016).

Resulta necesario mencionar dos aspectos importantes en los estudios de localismo; los cinco estudios identificados son de corte cualitativo y utilizan como técnicas para la recopilación de información la entrevista (en todos los casos) y la observación participante (en cuatro de ellos). Además, todos los estudios son de caso: Illawarra en Australia, San Pablo en Brasil, Nicaragua, Cornwall en Reino Unido y Pavones, en Costa Rica.

Por otro lado, se debe mencionar que los impactos económicos del turismo de surf es uno de los temas más abordados por los investigadores en los textos revisados (Carvalho y Winden, 2018; Hritz y Franzidis, 2018; Usher y Kerstetter, 2014; Rendle y Rodwell, 2014); y todos ellos hacen alusión a la derrama económica del turista. Por ejemplo, Hritz y Franzidis (2018) se centran en el gasto del turista de surf y su repercusión en el destino turístico; en su estudio concluyen que los turistas con más años de experiencia tienden a generar más derrama económica.

Por su parte, en la investigación realizada en Las Salinas, Nicaragua, Usher y Kerstetter (2014) reconocen que el turismo de surf tiene la capacidad de generar ingresos económicos en las localidades, pero esto no necesariamente se traduce en grandes magnitudes debido a que en el caso de estudio se comprobó que el turismo de surf no ha tenido la capacidad de contribuir a la mejora de la calidad de vida de la población. Por el lado contrario, el estudio de Towner y Davies (2018) en Mentawai, reconoce que dicho segmento turístico sí ha contribuido a dicha mejora de la comunidad, a la generación de empleos y a brindar la oportunidad de poder aprender inglés. Sin embargo, la población también ha relacionado este tipo turismo 
con temas como alcohol, drogas y el comportamiento irrespetuoso de los turistas de surf. Otro estudio similar al anterior es el Krause (2012), en Costa Rica.

De particular importancia resulta el estudio de Towner y Orams (2016), el cual aborda los impactos del turismo de surf y su relación con la sostenibilidad en las Islas Mentawai, Indonesia. Este estudio concluye, a través de la voz de los operadores de turismo de surf, que existen opiniones opuestas entre éstos sobre si los centros turísticos son o no sostenibles. Un hallazgo clave de esta investigación que se reportó, fue que los operadores consideraron que el turismo de surf ha alterado dramáticamente al tradicional pueblo Mentawai de Katiet contribuyendo a la producción de impactos socio-culturales adversos en la comunidad local.

Con base en lo antes expuesto se destaca el interés que ha recibido el localismo por parte de los investigadores como tópico central de investigación. En cuanto a los impactos económicos del turismo de surf, resalta la diversidad de resultados presentados por los investigadores, pues si bien resulta evidente que este tipo de turismo genera una derrama económica en los destinos de acogida, ésta no necesariamente se ve reflejada en la gran mayoría de los habitantes. De particular importancia resulta mencionar que en un solo estudio (Towner y Davies, 2018) se reconoce que el turismo de surf genera costos y beneficios específicos, por lo que se considera importante que más estudios se realicen bajo esta línea de investigación, pues si bien se ha señalado antes el apego que este segmento de turistas tiene por el cuidado del medio ambiente, no se puede dejar de lado que debido a su estilo de vida y comportamiento específico tienen incidencia en la comunidad que tal vez no está resultando positiva en todos los casos.

\subsubsection{Identidad y género en el turismo de surf}

Esta línea de investigación fue conformada a partir de la identificación de 8 artículos (9.87\%), que fueron divididos en dos grandes grupos: 1) los destinados al género a partir del papel y retos de las mujeres en el surf y 2) la contribución del surf a la generación de la identidad de los surfistas, tanto de hombres como de mujeres. Resulta importante señalar que 2017 fue el año cuando se publicaron la mayor cantidad de artículos bajo esta temática: 4 en total, lo que indica una tendencia entre los autores reportados.

Si bien la mayoría de los turistas surfistas suelen ser hombres, se ha identificado un incremento importante en la participación de las mujeres en este deporte. De hecho, el primer grupo de publicaciones identificadas resalta el tema de género y toma como referencia central a la mujer surfista. Entre los artículos revisados se encuentran el de Fendt y Wilson (2012) y el de Olive (2016); ambos textos muestran los retos y tensiones que las mujeres atraviesan al incursionar en un deporte que en su mayoría es y ha sido practicado por los hombres, destacando las restricciones sociales y culturales por las que han tenido que pasar para llevar a cabo el surf. Sin embargo, en su investigación, Fendt y Wilson (2012) destacan que las mujeres mantienen una actitud positiva hacia dicho deporte, mientras que Olive (2016) señala que ellas se suelen apoyar, logrando así hacer más significativa la práctica del surf.

Por su parte, Olive, et. al. (2015) dando voz a las mujeres, estudian cuáles son los cambios experimentados en ellas a partir de la práctica del surf, destacando cómo este deporte 
les ha permitido empoderarse en una destreza dominada por hombres. Finalmente, de particular importancia resulta el estudio de Fendt, et. al. (2014), en el cual a través del uso de la fenomenología a partir del ocio se reportan las experiencias vividas por las mujeres a través del surf.

En relación con los estudios de cómo influye el surf en la identidad, se identificaron cuatro investigaciones: Wheaton (2017a), Usher (2017); Wheaton (2017b); Usher y Kerstetter (2015b). De particular importancia resultan ser las publicaciones de Wheaton (2017a, 2017b) entre surfistas hombres y mujeres de mediana edad de Reino Unido, en las cuales se presenta cómo la edad impacta en la identidad, la movilidad del surf y las experiencias de los surfistas a través del envejecimiento. Ambos documentos destacan las formas en que los surfistas mayores se reproducen y desafían los discursos dominantes sobre el envejecimiento, la actividad física y la encarnación, y cómo prevén su futuro deportivo, negociando el envejecimiento a través del surf. Otro estudio elaborado bajo esta temática es el de Usher y Kerstetter (2015b) en Nicaragua, en el cual las autoras abordan el tema del significado real de ser un surfista local; para ello examinan la construcción de la identidad de los nicaragüenses como surfistas locales tomando en cuenta la presencia de surfistas extranjeros residentes o bien, de los surfistas extranjeros en su calidad de turistas.

A modo de dar continuidad a su estudio, pero esta vez en Costa Rica, Usher (2017) estudia cómo los surfistas expatriados en dicho país construyen su identidad como deportistas locales; esto como resultado del tiempo que han vivido y surfeado en la localidad de Pavones. En contraparte, para algunos costarricenses los surfistas con esta condición migratoria no son considerados locales. Finalmente, bajo la misma línea, pero con temas diferentes a los anteriores, destacan el estudio de Andrieu, et. al. (2017) con el tema de naturismo y la práctica del surf al desnudo en la playa de Tambaba en João Pessoa, Paraiba, Brasil. Así como el trabajo de Beaumont y Brown (2018) en Cornwall, Reino Unido que se enfoca en las interacciones entre los surfistas locales con la comunidad rural, y de qué manera este grupo podría contribuir a la formación, el mantenimiento y la identidad de esa comunidad rural más amplia.

Dado la naturaleza del tema, la mayoría de estas investigaciones emplean el enfoque cualitativo, haciendo uso de la etnografía como una de las principales técnicas para recabar información. Si bien el número de estudios bajo esta línea de investigación es escaso, se reconoce su importancia en el abordaje de temas relacionados con el género y su relación con las mujeres surfistas. Estos textos resultan de gran relevancia en tanto que el surf es en mayoría llevado a cabo por hombres por el esfuerzo y los riesgos que implica, pero la creciente participación de las mujeres en el mismo resulta ser una forma de romper ciertos paradigmas en relación con lo que se sabe del surf y su práctica.

\subsubsection{Historia del surf y los stakeholder}

En este apartado se presentan dos temáticas predominantes relativas a esta línea de investigación: la historia del surf y de los stakeholders, ambos vinculados con el desarrollo de dicho deporte. El primero de ellos se relaciona con la historia del surf en algunos destinos, reportándose datos generales en torno a ello (Esparza, 2017, 2014; Orams y Towner, 2012); o 
bien, información centrada en estudios de caso: Doering en Miyazaki, Japón (2018); Esparza en Cádiz y Málaga en Andalucía (2015); Esparza en España (2013; 2016); Thompson en KwaZuluNatal (Zululandia), Sudáfrica (2014); y el de Canniford y Karababa sobre el significado y usos del discurso primitivo del consumidor a través del surf indígena hawaiano (2013). En este mismo grupo se contempla el artículo publicado por Del Giudice y Giraldo (2014), que si bien, no es un artículo que trate únicamente sobre la historia del surf, si puede ser un documento que ayude a comprender el surf y su dinámica social. Todos estos artículos en conjunto contribuyen a la compresión del surgimiento del surf alrededor del mundo, si bien, resultan ser pocos los identificados dentro de este periodo de estudio, se debe tomar en cuenta que dentro de las investigaciones de Martin y Assenov $(2012,2011)$ fueron de las principales, pues fue en décadas pasadas recientes donde el tema recibió mayor atención. De entre este breve conjunto de artículos, es necesario resaltar las publicaciones de Esparza (2017, 2016, 2015, 2014, 2013), pues por un lado se han centrado en la historia y desarrollo del surf, pero también, es uno de los pocos investigadores que ha escrito sobre el surf en países de habla hispana.

El segundo enfoque de la línea está conformado por estudios relacionados con los stakehokders, es decir, con todos aquellos grupos que en un momento están involucrados en el turismo de surf y que impulsan su desarrollo. Únicamente cinco fueron las investigaciones encontradas sobre este tema, todas ellas realizadas entre 2015 a 2018; sin embargo, destacan tres investigaciones realizadas por Tower: en la primera de ellas, relacionada con las islas Mentawai (Towner, 2018), el autor examina la colaboración de las partes interesadas en la industria del turismo de surf mediante la exploración de múltiples perspectivas. En los resultados de la investigación se señala que la corrupción, la desconfianza tradicional hacia el gobierno y la falta de una iniciativa de colaboración organizada, se consideran las principales barreras para una colaboración efectiva en la industria del turismo de surf en las islas estudiadas.

En las otras dos investigaciones, Towner (2015) y Towner y Milne (2017), se reconoce la complejidad de estudiar en conjunto las diversas partes interesadas en el turismo de surf (comunidad, gobierno, operadores turísticos y turistas), pues cada una de ellas tiene percepciones distintas en torno al mismo. En los resultados se reporta que los funcionarios gubernamentales y los operadores de barcos de alquiler pensaron que la industria del turismo de surf se había desarrollado de una manera insostenible, atribuyendo esto a la corrupción gubernamental, que no gestionaba la región de manera efectiva. Por su parte, los operadores de resorts percibieron que la industria del turismo de surf se había desarrollado de manera sostenible y se encontraba dentro de su capacidad de carga. Finalmente, los miembros de la comunidad local entrevistados pensaron que los turistas eran una fuerza muy positiva en sus comunidades debido a los beneficios económicos producidos por sus viajes. Es a partir de lo anterior que Towner y Milne (2017) señalan la necesidad de lograr un desarrollo más sostenible en las Islas a través de una gobernanza más operativa y una participación activa por parte del gobierno.

Otro estudio parecido al anterior es el realizado por Buckley, et. al. (2017), en las islas Maldivas; en dicha investigación se da apertura a un grupo mayor de stakeholders (trabajadores de complejos turísticos, turistas de surf que prefieren complejos turísticos, empleados en 
barcos de alquiler, surfistas que utilizan botes, empresas locales relacionadas con el surf, surfistas independientes que emplean dichas empresas y surfistas residentes locales). También se evidencia lo complejo de este tipo de estudios, pues los intereses involucrados son diversos. El trabajo de Ratten (2018) únicamente estudia las intenciones empresariales de los turistas surfistas en relación con un evento de surf. Finalmente, Sariego y Moreno (2015) centrándose en el Municipio de Ribamontán al Mar, España presentan un análisis, apoyado en cifras, que demuestran y justifican la importancia del turismo de surf en el destino.

Los estudios de stakeholders identificados, dejan ver cómo cada una de las partes que interviene en la práctica del turismo de surf presenta realidades e intereses diferentes, situación que vuelve complejo su análisis de manera conjunta. Sin embargo, resulta necesario intentar conocerlas y comprenderlas pues a partir de ello se pueden atender, o bien, dilucidar si hubiese una concepción errónea.

\section{CONCLUSIONES}

Resulta evidente tomando en cuenta los 42 artículos identificados por Martin y Assenov (2012) el incremento en el número de artículos publicados sobre turismo de surf en el periodo considerado en este estudio, si bien, en esta investigación se incluyeron publicaciones en español, en realidad no tienen gran participación sobre el total, únicamente 11, en este sentido resultan necesarias más investigaciones en este idioma que brinden un panorama sobre el turismo de surf, pues como se ha señalado a lo largo de este texto, el tópico ha sido encabezado por países anglosajones, Estados Unidos y Australia. Si bien, se han realizado algunas investigaciones en países en desarrollo, en realidad poco se sabe sobre el turismo de surf en este tipo de destinos. Resulta evidente que el estudio permite visualizar una tendencia en el incremento de las investigaciones del turismo de surf, sin embargo, aún existen muchos vacíos de conocimiento que exigen mayores investigaciones a partir de diferentes disciplinas y temáticas. Asimismo, se reconoce la importancia de realizar estudios a partir de enfoques teóricos que permitan analizar los hallazgos de las investigaciones con teorías o conceptos provenientes de diversas disciplinas sociales, pues en el presente estudio, poco se observó de ello.

En este estudio se identificaron cinco líneas principales de investigación; 1) El surfista; 2) Turismo de surf, desarrollo local y sustentabilidad; 3) Impactos del turismo de surf; 4) Identidad y género en el turismo de surf; 5) Historia y desarrollo del surf y de los stakeholders, sin duda alguna todas ellas contribuyen de forma considerable al periodo progresivo -the progressive period-identificado por Martín y Assenov (2012). De las cinco líneas identificadas, la uno y la dos, son las que mayor atención ha recibido por parte de los investigadores. Es de destacar el apego hacia la metodología cuantitativa en los estudios identificados en línea del surfista y la cualitativa en el caso de la línea turismo de surf, desarrollo local y sustentabilidad. Por su parte las tres líneas restantes muestran un uso más equilibrado entre ambas metodologías, haciendo gran uso de la etnografía, la entrevista y la observación participante para recabar información. Quizás dicha inclinación se debe a que en estas líneas más que conocer sobre el turismo de surf, se busca comprenderlo. 
Tomando en cuenta el incremento en la investigación sobre turismo de surf, se sugiere ahondar en cada una de las líneas de investigación identificadas, así como en aquellas que actualmente no han despertado el interés de los investigadores. En el caso del surfista se sugieren más estudios sobre los perfiles, las motivaciones, las actitudes y comportamientos en los destinos. En cuanto a la línea de desarrollo local y sustentabilidad se recomienda tomar en cuenta que los spots son sensibles a los cambios del entorno natural, por lo tanto, todos aquellos interesados y beneficiados de estos deberían realizar un trabajo en conjunto para su protección y cuidado. Más investigaciones en esta línea podrían ayudar al gobierno y a las comunidades a un manejo y una gestión más sostenible de dichos recursos. Asimismo, se recomienda involucrar a los turistas surfistas en ello, así como hacer uso de su conocimiento sobre las características geofísicas de los puntos de surf.

En la línea de impactos del turismo, se recomienda realizar estudios sobre los impactos económicos del turismo de surf, pero centrándose en el análisis de su incidencia en la comunidad residente y en su calidad de vida. También, resulta necesario realizar más investigaciones sobre los impactos socioculturales y ambientales que el turismo de surf y el surfista mismo, generan en la comunidad. Lo anterior se recomienda en la medida que, entre los estudios identificados, si bien se menciona como el turismo de surf ha impactado negativamente en algunos destinos, como tal no existe gran cantidad de investigaciones al respecto.

En cuanto a la línea de identidad y género se recomienda hacer más estudios sobre la comunidad de surfistas para tener un mayor entendimiento de sus valores, significados del mar y la naturaleza. Asimismo, se recomienda centrar esfuerzos en estudios sobre las mujeres surfistas y su papel dentro de dicho deporte, sus retos, experiencias de viaje, empoderamiento o desigualdades. Por último, como se señaló antes, la línea de investigación de historia y desarrollo del surf y de los stakeholders, resulta ser fundamental para aquellos destinos de surf que no han sido atendidos al día de hoy, pues si bien en países como Estado Unidos, Australia, Nueva Zelanda, Reino Unido, Brasil, entre otros, dichas investigaciones ya han sido realizadas, existen otros donde el conocimiento es casi nulo.

Este estudio puede servir de guía a investigadores y estudiantes para futuros temas de investigación. Si bien, este documento se centró únicamente en los artículos científicos publicados sobre turismo de surf, se recomienda dar seguimiento al estudio de Martín y Assenov (2012) en el cual se incluyan diversas fuentes para hacer un estudio más completo que evidentemente, puede generar un cambio en los resultados aquí presentes. Además, se recomienda hacer un estudio donde se tomen artículos científicos publicados en otras lenguas, pues eso hará los resultados más sólidos. Atendiendo el señalamiento de Martin y Assenov $(2011 ; 2012)$ se incentiva a que más estudios de grado sean realizados sobre el turismo de surf. Finalmente, los autores de este escrito están de acuerdo en señalar que el turismo de surf hoy en día, ha pasado de su génesis (Martín y Assenov; 2011; 2012), a formar como tal un campo de estudios en crecimiento y desarrollo, pues la revisión realizada permite una mayor compresión del surf, del turismo de surf, del surfista y de una parte de los involucrados en su práctica. 
Agradecimientos

Los autores del presente documento agradecen a todos aquellos autores que compartieron sus publicaciones para hacer de este trabajo un documento los más completo posible.

\section{REFERENCIAS BIBLIOGRÁFICAS}

Aaron, E., y Wayne, S. (2013). Assessing the Potential for Surf Break Co-Management: Evidence from New Zealand. Coastal Management, 41 (6), 537-560. Doi: 10.1080/08920753.2013.842681

Andrieu, B., Da Nóbrega, T., y Sirost, O. (2017). Naked surfing: A Brazilian example of body ecology, Society and Leisure, 40 (1), 76-84. Doi: 10.1080/07053436.2017.1282015

Barbieri, C., y Sotomayor, S. (2013). Surf travel behavior and destination preferences: An application of the Serious Leisure Inventory and Measure. Tourism Management, 35,111-121. Doi: https://doi.org/10.1016/j.tourman.2012.06.005

Barbieri, C., Henderson, K., y Santos, C. (2014). Exploring memorable surfing trips. Annals of Tourism Research, 48, 277-280. Doi: http://dx.doi.org/10.1016/j.annals.2014.06.008

Beaumont, E., y Brown, D. (2015). 'a Local Surfer, Always a Local Surfer': Local Surfing Careers in a Southwest English Village. Leisure Sciences, 37 (1), 68-86. Doi: 10.1080/01490400.2014.952462

Beaumont, E., y Brown, D. (2016). 'It's not something I'm proud of but it's ... just how I feel': local surfer perspectives of localism. Leisure Studies, 35 (3), 278-295. Doi: 10.1080/02614367.2014.962586

Beaumont, E., y Brown, D. (2018). 'It's the sea and the beach more than anything for me': Local surfer's and the construction of community and communitas in a rural Cornish seaside village. Journal of Rural Studies, 59, 58-66.

Brochado, A., Stoleriu, O., y Lupu, C. (2018). Surf camp experiences. Journal of Sport \& Tourism, 22 (1), 21-41. Doi: 10.1080/14775085.2018.1430609

Buckley, R. (2002). Surf Tourism and Sustainable Development in Indo-Pacific Islands. The Industry and the Islands. Journal of Sustainable Tourism, 10 (5), 405- 424. Doi: 10.1080/09669580208667176

Buckley, R., Guitart, D., y Shakeel, A. (2017). Contested surf tourism resources in the Maldives. Annals of Tourism Research, 64, 185-199. Doi: https://doi.org/10.1016/i. annals.2017.03.005

Canniford, R., y Karababa, E. (2013). Partly primitive: discursive constructions of the domestic surfer. Consumption Markets \& Culture, 16 (2), 119-144. Doi: 10.1080/10253866.2012.662818

Carvalho, L., y Winden, W. (2018). Making waves: the valuation of innovations in San Sebastian's surf economy. European Planning Studies, 26 (1), 75-93. Doi:10.1080/09654313.2017 .1372369

D’Ambrosio, L. (2017). La experimentación perceptual de la costa y el mar: un estudio con surfistas, biologos y pescadores artesanales. Pelotas, 5 (1), 29-55. 
Del Giudice, O., y Giraldo, M. (2014). Análisis descriptivo de la dinámica del surf como práctica social. Telos, Revista de Estudios Interdisciplinarios en Ciencias Sociales, 16 (2), 300314. Recuperado de: https://www.redalyc.org/articulo.oa?id=99331125009

Doering, A. (2018). Mobilising Stoke: A Genealogy of Surf Tourism Development in Miyazaki, Japan. Tourism Planning and Development, 15 (1), 68-81. Doi: https://doi.org/10.1080 $\angle 21568316.2017 .1313772$

Dolnicar, S., y Fluker, M. (3003). Who's Riding the Wave? An Investigation Into Demographic and Psychographic Characteristics of Surf Tourists, 13th International Research Conference for the Council for Australian University Tourism and Hospitality Education (CAUTHE 2003).

Eka, A. (2012). Dimensions of Community-Based Tourism: A Case Of Surf Community In Bali, Indonesia. Journal of Tourism Research, 5, 38-46. Recuperado de: http://www.jotr.eu/ pdf files/V5.pdf\#page $=36$

Esparza, D. (2011). De Hawaii al Mediterráneo: La génesis del surf en España. Revista Internacional de Ciencias del Deporte, 7 (6), 370-383. Recuperado de: https://www. cafyd.com/REVISTA/ojs/index.php/ricyde/article/view/443

Esparza, D. (2013). Historia y Deporte: Los orígenes del surf a través de la prensa Española. Romanica Olomucensia, 25 (1), 47-59. Recuperado de: https://dialnet.unirioja.es/ servlet/articulo?codigo $=4460034$

Esparza, D. (2014). Un hombre llamado pez: la historia de Duke Kahanamoku, el nadador más rápido del mundo y el padre del surf moderno. RICYDE. Revista Internacional de Ciencias del Deporte, 10 (38), 383-386. Recuperado de: https://www.cafyd.com/REVISTA/ojs/ index.php/ricyde/article/view/799

Esparza, D. (2015). Hacia una historia del surf en Andalucía: Génesis consolidación del surf en Cádiz y Málaga. Materiales para la Historia del Deporte, 13, 47-62. Recuperado de: $\quad$ https://www.upo.es/revistas/index.php/materiales historia_deporte/article/ view/1327

Esparza, D. (2016). Towards a theory of surfing expansion: The beginnings of surfing as a case study. Ricyde: Revista Internacional de Ciencias del Deporte, 44 (12), 199-215. Recuperado de: https://www.cafyd.com/REVISTA/ojs/index.php/ricyde/article/ view/957

Esparza, D., (2017). Reconsiderando las fuentes para el estudio del surf Arcaico: Polinesia, China, Perú y África Occidental. Materiales para la historia del deporte, 15, 193-213. Recuperado de: https://www.upo.es/revistas/index.php/materiales historia deporte/ article/view/2602

Fendt, I., y Wilson, E. (2012). 'I just push through the barriers because I live for surfing' how women negotiate their constraints to surf tourism. Annals of Leisure Research, 151, 4-18.

Fendt, L., Wilson, E., Jenkins, J., Dimmock, K., y Weeks, P. (2014). Presenting phenomenology: faithfully recreating the lived experiences of Surfer Girls. Annals of Leisure Research, 17 (4), 398-416. Doi: 10.1080/11745398.2014.938668

Frank, F., Pintassilgo, P., y Pinto, P. (2015). Environmental Awareness of Surf Tourists: A Case Study in the Algarve. Journal of Spatial and Organizational Dynamics, 3 (2), 102-113. Recuperado de: https://ideas.repec.org/a/ris/ispord/0047.html 
Frontrodona, M. (13 de noviembre de 2016). "Cinco estudios sobre surf dejan datos realmente curiosos". Diario AS Espana-Diario Online de deporte. Recuperado de: https://as.com/ deportes accion/2016/09/24/agua/1474715400_951492.html

Guibert, G., y Taunay, B. (2013). From Political Pressure to Cultural Constraints: The Prime Dissemination of Surfing in Hainan. Journal of China Tourism Research, 9 (3), 365-380. Doi: 10.1080/19388160.2013.812903

Hritz, N., y Franzidis, A. (2018). Exploring the economic significance of the surf tourism market by experience level. Journal of Destination Marketing \& Management, 7, 164-169. Doi: 10.1016/j.jdmm.2016.09.009

ISA. (16 de Marzo de 2018). Obtenido de International Surfing Association: https://www. isasurf.org/es/international-olympic-committee-and-isa-confirm-qualification-process-for-surfing-competition-at-tokyo-2020/

Jopp, R., De Lacy, T., Mair, J., y Fluker, M. (2013). Using a Regional Tourism Adaptation Framework to Determine Climate Change Adaptation Options for Victoria's Surf Coast. Asia Pacific Journal of Tourism Research, 18 (1), 144-164. Doi: 10.1080/10941665.2012.688515

Krause, S. (2012). Pilgramage to the playas Surf Tourism in Costa Rica. Anthropology in Action, 19, (3), 37-48. Doi: 10.3167/aia.2012.190304

Kumara, W., Silva, M., y Madusanka, R. (2018). The lack of participation for surfing by Sri Lankans: Surfing Areas. Journal of Sports and Physical Education, 5 (5), 1-7. Doi:10.9790/6737-05050107

Larson, L., Usher, L., y Chapmon, T. (2018). Surfers as Environmental Stewards: Understanding Place-protecting Behavior at Cape Hatteras National Seashore. Leisure Sciences, 40 (5), 442-465. Doi: DOI: 10.1080/01490400.2017.1305306

Mach, L., y Ponting, J. (2018). Governmentality and surf tourism destination governance. Journal of Sustainable Tourism, 26 (11), 1845-1862. Doi: 10.1080/09669582.2018.1513008

Mach, L., Ponting, J., Brown, J., y Savage, J. (2018). Riding waves of intra-seasonal demand in surf tourism: analysing the nexus of seasonality and 21st century surf forecasting technology. Annals of Leisure Research, DOI: 10.1080/11745398.2018.1491801.

Machado, V., Carrasco, P., Pinto, J., Patrícia, A., y Gouveia, D. (2018). Governing Locally for Sustainability: Public and Private Organizations' Perspective in Surf Tourism at Aljezur, Costa Vicentina, Portugal. Tourism Planning y Development, 15 (6), 692-704. Doi:10.10 80/21568316.2017.1415958

Martín, R. y Luque-Gil, A. (2014). Propuesta de diversificación de productos en destinos consolidados: el turismo de surf en la Costa del Sol Occidental. Estudios Turísticos, 199, 63-88. Recuperado de: https://dialnet.unirioja.es/servlet/articulo?codigo $=4829272$

Martin, S., y Assenov, I., (2012). The genesis of a new body of sport tourism literature: a systematic review of surf tourism research (1997-2011). Journal of Sport \& Tourism, 17 (4), 257-287. Doi: 10.1080/14775085.2013.766528

Martin, S., y Assenov, I. (2014a). Developing a Surf Resource Sustainability Index as a Global Model for Surf Beach Conservation and Tourism Research. Asia Pacific Journal of Tourism Research, 19 (7), 760-792. Doi: 10.1080/10941665.2013.806942

Martin, S., y Assenov, I. (2014b). Investigating the Importance of Surf Resource Sustainability Indicators: Stakeholder Perspectives for Surf Tourism Planning and Development. Tourism Planning \& Development, 11 (2), 127-148. Doi: 10.1080/21568316.2013.864990 
Martin, S., y Assenov, I. (2011). A statistical analysis of surf tourism research literature. CD Proceedings of the 4th Annual PSU Research Conference: Multidisciplinary Studies on Sustainable Development (p. 57). Prince of Songkla University, Phuket, Thailand, November 16-18.

Martins, M. (2014). Territorial disputes, identity conflicts, and violence in surfing. Motriz, Rio Claro, 20 (1),16-25. Doi: http://dx.doi.org/10.1590/S1980-65742014000100003

Martin, S., y Ritchie, R., (2018). A Social Science Index and Conceptual Framework for Assigning Weights in Surf Tourism Planning and Development. Tourism Planning \& Development, 16 (3), 281-303. Doi: 10.1080/21568316.2018.1470999

Mills, B., y Cummins, A. (2013). The economic impact of domestic surfing on the United Kingdom. Reino Unido: Surfers Against Sewage.

Nazlı M., y Musal R. (2018). Surf Tourism Development: Perspectives In Alaçat. Surf Spot, 6 (2), 390-409.

O'Brien, D., y Ponting, J. (2013). Sustainable Surf Tourism: A Community Centered Approach in Papua New Guinea. Journal of Sport Management, 27 (2), 158-172.

Olive, R., (2016). Going surfing/doing research: learning how to negotiate cultural politics from women who surf, Continuum: Journal of Media \& Cultural Studies, 30 (2), 171-182. Doi: 10.1080/10304312.2016.1143199

Olive, R., McCuaig, L., y Phillips, M. (2015). Women's recreational surfing a patronising experience, Sport. Education and Society, 202, 258-276.

Orams, M., y Towner, N. (2012). Riding the Wave: History, Definitions, and a Proposed Typology of Surf-riding Tourism. Tourism in Marine Environments, 8 (4), 173-188. https://doi.org $\angle 10.3727 / 154427313 \times 13631129554901$

Orchard, S. (2017). Lessons for the design of surf resource protection - The Australasian experience. Ocean \& coastal management, 148, 9-112. Doi: https://doi.org/10.1016/j. ocecoaman.2017.07.019

Ponting, J. (2008). Consuming Nirvana: An exploration of surfing tourist space (Doctoral dissertation). University of Technology, Sydney.

Ponting, J., (2014). Comparing modes of surf tourism delivery in the Maldives, Annals of Tourism Research, 46,163-184. DOI: 10.1016/j.annals.2014.01.001

Ponting, J., y McDonald, M. (2013). Performance, agency and change in surfing tourist space, Annals of Tourism Research, 43, 415-434. Doi: https://doi.org/10.1016/i. annals.2013.06.006

Ponting, J., y O'Brien, D. (2014). Liberalizing Nirvana: an analysis of the consequences of common pool resource deregulation for the sustainability of Fiji's surf tourism industry. Journal of Sustainable Tourism, 22 (3), 384-402. Doi: 10.1080/09669582.2013.819879

Ponting, J., y O'Brien D. (2015). Regulating "Nirvana": Sustainable surf tourism in a climate of increasing regulation. Sport Management Review, 18, (1), 99-110. Doi: https://doi. org/10.1016/j.smr.2014.07.004

Porter, B., Orams, M., y Lück, M., (2015). Surf-riding tourism in coastal fishing communities: A comparative case study of two projects from the Philippines. Ocean \& Coastal Management, 116, 169-176. Doi: https://doi.org/10.1016/i.ocecoaman.2015.07.015

Porter, B., y Usher, L. (2018). Sole surfers?: exploring family status and travel behaviour among surf travelers. Annals of Leisure Research, DOI: 10.1080/11745398.2018.1484782 
Portugal, A., Campos, F., Melo, R., y Lourenço, F. (2017). Understanding the relation between serious surfing, surfing profile, surf travel behavior and destination attributes preferences. European Journal of Tourism Research, 16, 57-73. Recuperado de: https://www. researchgate.net/publication/317904681 Understanding the relation between serious surfing surfing profile surf travel behavior and destination attributes preferences

Ratten, V. (2018). "Entrepreneurial intentions of surf tourists". Tourism Review, 73 (2), 262276. Recuperado de: https://www.emerald.com/insight/content/doi/10.1108/ TR-05-2017-0095/full/html

Reineman, D. (2016). The utility of surfers' wave knowledge for coastal management. Marine Policy, 67, 139-147.

Reineman, D., y Ardoin, N. (2018). Sustainable tourism and the management of nearshore coastal places: place attachment and disruption to surf-spots. Journal of Sustainable Tourism, 26 (2), 325-340. Doi: 10.1080/09669582.2017.1352590

Rivera, M. (2016). Paisaje, patrimonio y turismo de surf: factores de atracción y motivación en el Parque Natural del Estrecho, España. Cuadernos de Turismo, 37, 351-76. Doi: http:// dx.doi.org/10.6018/turismo.37.256271

Santander, P. (2011). Por qué y cómo hacer análisis del discurso. Cinta Moebio: Revista de Epistemología de Ciencias Sociales, 41, 207-224. Recuperado de: https://dialnet.unirioja.es/servlet/articulo?codigo $=3709384$

Sariego, I., y Moreno, A. (2015). El desarrollo turístico y territorial Baado en el surf: Ribamontán al Mar, "surf a toda costa". Estudios Turísticos. 205, 119-138. Recuperado de: https:// dialnet.unirioja.es/servlet/articulo?codigo $=5516663$

Scott, S., y Rogers, S. (2018). Surf's up? How does water quality risk impact surfer decisions?. Ocean \& Coastal Management, 151, (1), 53-60. Doi: https://doi.org/10.1016/j. ocecoaman.2017.10.025

Silva, C., Seabra, C., Abrantes, J., y Faias, C. (2014). Motivations, involvement and place-attachment in surf tourism. Revista Turismo \& Desenvolvimiento, 21 (22), 51-53. Recuperado de: https://dialnet.unirioja.es/servlet/articulo?codigo $=5436315$

Sotomayor, S., y Barbieri, C. (2016). An Exploratory Examination of Serious Surfers: Implications for the Surf Tourism Industry. Internacional Journal of Tourism Research, 18, (1), 62-73. Doi: https://doi.org/10.1002/itr.2033

Thompson, G. (2014). Otelo Burning and Zulu surfing histories. Journal of African Cultural Studies, 26 (3), 324-340. Doi: 10.1080/13696815.2014.940037

Tien-Ming, C., y Sheng-Hshiung, T. (2012). The relationship between serious leisure characteristics and recreation involvement: a case study of Taiwan's surfing activities. Leisure Studies, 31 (1), 53-68. Doi: 10.1080/02614367.2011.568066

Tien-Ming, C., Y Chiang-Chuan, L. (2015). The Causal Relationships among Recreational Involvement, Flow Experience, and Well-being for Surfing Activities. Asia Pacific Journal of Tourism Research, 20 (1), 1486-1504. Doi: 10.1080/10941665.2014.999099

Tourism New South Wales (2009) Tourism NSW's Action Plan to Consolidate the State's Position as Australia's Premier Surf Destination: Catching the Wave (Sydney: New South Wales Government and Author). 
Towner, N. (2015). Surf tourism and sustainable community development in the Mentawai Islands, Indonesia: a multiple stakeholder perspective. European Journal of Research, 11, 166-170. Recuperado de: https://www.questia.com/read/1P3-3864677251/ surf-tourism-and-sustainable-community-development

Towner, N. (2016a). Community participation and emerging surfing tourism destinations: a case study of the Mentawai Islands. Journal of Sport \& Tourism, (20) 1, 1-19. Doi: 10.1080/14775085.2016.1151819

Towner, N. (2016b). Searching for the perfect wave: Profiling surf tourists who visit the Mentawai Islands. Journal of Hospitality and Tourism Management, 26, 63-71. Recuperado de: https://doi.org/10.1016/i.jhtm.2015.11.003

Towner, N. (2016c). How to manage the perfect wave: Surfing tourism management in the Mentawai Islands, Indonesia. Ocean \& Coastal Management, 119, 217-226. Doi: https://doi.org/10.1016/j.ocecoaman.2015.10.016

Towner, N. (2018). Surfing tourism and local stakeholder collaboration. Journal of Ecotourism, 17 (3), 268-286. Doi:10.1080/14724049.2018.1503503

Towner, N., y Orams, M. (2016). Perceptions of surfing tourism operators regarding sustainable tourism development in the Mentawai Islands, Indonesia. Asia Pacific Journal of Tourism Research, 21 (11) 1258-1273. Doi: 10.1080/10941665.2016.1140663

Towner, N., y Milne, S. (2017). Sustainable Surfing Tourism Development in the Mentawai Islands, Indonesia: Local Stakeholder Perspectives. Tourism Planning \& Development, 14 (4), 503-526. Doi: 10.1080/21568316.2017.1287122

Towner, N., y Davies, S. (2018) Surfing tourism and community in Indonesia, Journal of Tourism and Cultural Change, DOI: 10.1080/14766825.2018.1457036

Usher, L. (2017). "Foreign locals": transnationalism, expatriates, and surfer identity in Costa Rica. Journal of Sport \& Social Issues, 41 (3), 212-238. Doi: 10.1177/0193723517705542

Usher, L., y Kerstetter, D. (2014). Residents' perceptions of quality of life in a surf tourism destination: A case study of Las Salinas, Nicaragua. Progress in Development Studies. 14 (4), 321-333. Doi: https://doi.org/10.1177/1464993414521525

Usher, L., y Kerstetter, D. (2015a). Re-defining localism: an ethnography of human territoriality in the surf. Int. J. Tourism Anthropology, (4), 3, 286-302. Doi: OI: 10.1504/ IJTA.2015.071930

Usher, L., y Kerstetter, D. (2015b). Surfistas Locales: Transnationalism and the Construction of Surfer Identity in Nicaragua. Journal of Sport and Social, 39, (6), 455-479. Doi: https:// doi.org/10.1177/0193723515570674

Usher, L., y Gómez, E. (2016). Surf localism in Costa Rica: exploring territoriality among Costa Rican and foreign resident surfers. Journal of Sport \& Tourism, 20, (3-4), 195-216. Doi: 10.1080/14775085.2016.1164068

Usher, L., Goff, J., y Gómez, E. (2016). Exploring surfers' perceptions of municipal regulations using grounded theory. Annals of Leisure Research, 19 (1), 98-116. Doi: 10.1080/11745398.2015.1066260

Usher, L., y Gómez, E. (2017). Managing Stoke: Crowding, Conflicts, and Coping Among Virginia Beach Surfers. Journal of Park \& Recreation Administration. 35 (2), 9-24. Doi: http:// dx.doi.org/10.18666/JPRA-2017-V35-12-7596 
Waitt, G., y Frazer, R. (2012). "The vibe" and "the glide": surf ing through the voices of longboarders. JournalofAustralianStudies, (36)3, 327-343. Doi:10.1080/14443058.2012.703685

Ware, D., (2017). Sustainable resolution of conflicts over coastal values: a case study of the Gold Coast Surf Management Plan. Australian Journal of Maritime \& Ocean Affairs, 9 (2), 68-80. Doi: 10.1080/18366503.2017.1278501

Wheaton, B., (2017a). Staying 'stoked': Surfing, ageing and post-youth identities. International Review for the Sociology of Sport. Doi: https://doi.org/10.1177/1012690217722522.

Wheaton, B., (2017b). Surfing through the life-course: silver surfers' negotiation of ageing, Annals of Leisure Research, 20 (1), 96-116. Doi: 10.1080/11745398.2016.1167610 